Article

\title{
Consistent DSC and TGA Methodology as Basis for the Measurement and Comparison of Thermo-Physical Properties of Phase Change Materials
}

\author{
Lukas Müller ${ }^{1, *}$, Gabriel Rubio-Pérez ${ }^{2}{ }^{(}$, Andreas Bach ${ }^{3}$, Natalia Muñoz-Rujas ${ }^{2}{ }^{\circledR}$, \\ Fernando Aguilar ${ }^{2}$ (I) and Jörg Worlitschek ${ }^{1}$ (D) \\ 1 Competence Centre Thermal Energy Storage (TES), Lucerne University of Applied Sciences and Arts, \\ 6048 Horw, Switzerland; joerg.worlitschek@hslu.ch \\ 2 Escuela Politécnica Superior, Universidad de Burgos, 09006 Burgos, Spain; grubio@ubu.es (G.R.-P.); \\ nmrujas@ubu.es (N.M.-R.); faguilar@ubu.es (F.A.) \\ 3 Business Unit Materials Characterization, Mettler Toledo GmbH, 8606 Nänikon, Switzerland; \\ andreas.bach@mt.com \\ * Correspondence: lukas.mueller@hslu.ch; Tel.: +41-(0)41-349-3707
}

Received: 11 September 2020; Accepted: 7 October 2020; Published: 10 October 2020

\begin{abstract}
Measuring thermo-physical properties of phase change materials (PCM) in a consistent and reliable manner is essential for system layout of thermal energy storages and correspondingly material selection. Only if basic properties are assessed in a comparable way a selection process leads to the top candidate for any given application and thus enhances market penetration of renewable energy sources coupled with thermal energy storage. In this study, we focus on differential scanning calorimetry (DSC) and thermogravimetric analysis (TGA) as basic assessment techniques and develop consistent measurement procedures to create a database with comparable results. We show consistency of the measured results through analysis of coefficient of variation $(\mathrm{CV})$, being in the mean $1.69 \%, 0.05 \%, 0.06 \%$ and $4.00 \%$ for enthalpy, melting onset, melting peak and maximum operating temperature, respectively. Overall, 23 PCM have been measured with the presented methodology, which was mainly possible due to the reduced measurement and preparation time per PCM compared to standard techniques, while achieving similar accuracy and precision.
\end{abstract}

Keywords: phase change material; latent heat storage; thermal energy storage; differential scanning calorimetry; thermogravimetric analysis; energy

\section{Introduction}

The current energy system transformation will lead to an increase of renewable power generation, of which wind and solar photovoltaic are considered to have major contributions. [1] Both technologies require storage concepts due to fluctuations in energy production. Such storage concepts can consist of a manifold of different technologies and types of energy stored. In moderate temperature climate zones, more than half of the energy end-use is governed by space heating [2], therefore, storing energy surplus when available as heat is logical. Furthermore, similar integration concepts can be used for storing cold, which will become a substantial need of the future due to the effects of climate change [2].

One particular technology for short-term heat/cold storage is known as latent thermal energy storage, a system based on a phase change material (PCM), a heat exchanger and a power source. The PCM stores large amounts of energy due to a phase change, commonly liquid-solid, at a 
specific temperature. In the last two decades, interest in PCM regarding properties, system integration and applications have risen substantially and generated over 4300 research publications [3].

Several different research tracks within the field of PCM development and characterization can be identified. They constitute the modification of PCM through the introduction of additives to overcome undesired properties, for example flammability in organic PCM [4], enhancing the thermal conductivity [5], specific heat capacity [6], or segregation in salt hydrates [7,8]. In addition, the development and integration of bio-based PCM [9-11] into several applications such as thermal regulation of buildings [12] or clothing [13].

A recent problem in the development of latent thermal energy storage systems is the variance in published basic thermo-physical properties, particularly melting point and enthalpy. This leads to complicated material selection processes due to uncertainty of the measured data. Therefore, a critical review of several sources or in-house measurements of these basic properties is usually required. The reason for this variance in data lies within the manifold of measurement techniques available and experimental parameters considered, complicating the comparability and selection of materials [14-17].

Published literature concerning standard measurement methodologies with specific focus on PCM is scarce. There are a number of standards describing differential scanning calorimetry, like DIN 53765 [18], ISO 11357-3 [19], ASTM E 793 [20], and ASTM D 3418 [21], which are either non-specific regarding sample material or for a specific group of materials (e.g. ASTM D 3418 is for polymers). Within those industry standards, the recommended heating rate is usually $10 \mathrm{~K} \mathrm{~min}^{-1}$ for dynamic mode. In PCM publications, several different heating/cooling rates are used, as low as 0.5 up to $10 \mathrm{~K} \mathrm{~min}^{-1}$. [22-26] Barreneche et al. have studied the difference of operating mode, i.e. dynamic or step mode. [27] They found dynamic mode to be suitable for the analysis of organic and inorganic PCM.

It is common in published literature to omit important data used during a study. The sample mass during an investigation is usually not reported in PCM studies. Castellón et al. have shown that the sample mass has an influence on the properties as well as the heating rate [28]. A standard for the measurement of properties of PCM was published by the RAL Institute in Germany, and a round robin test was carried out in the context of Task 42 from the IEA Solar Heating and Cooling Program [29-31]. These publications cover a wide range of elements that need to be taken into account, like influence of sample mass, heating/cooling rate or type of analysis. However, a detailed standard methodology that could be used as a research model in the laboratory is still lacking. Additionally, the preparation and measurement of this standard uses differential scanning calorimetry (DSC) measurements exclusively, resulting in a lack of variability of measured PCM. Furthermore, the change in heating/cooling rate per measured substance results in a limited comparability of properties.

Regarding thermogravimetric analysis (TGA) methodology, an even greater disagreement is found. Experimental parameters vary widely, the reported results are manifold, and therefore a comparison between different PCM is difficult to impossible. Some standards describing the thermogravimetric analysis do exist, for example, ASTM C 1872 [32], ISO 11358-1 [33] and ASTM E 1131 [34]. The first two are specific to a certain material group, while the latter is rather generic and vague. None of the above-mentioned standards suggest a method for analysis of PCM specific properties, thus we present a possible evaluation of the maximum operating temperature within this work.

In this work, we present two measurement protocols applicable to a variety of different materials, all of them used as PCM with melting points between $-50^{\circ} \mathrm{C}$ and $190^{\circ} \mathrm{C}$. Using consistent experimental parameters, we demonstrate a comparison of the resulting material properties for an accurate evaluation of application specific PCM. We present a set of PCM materials, their thermal characteristics and compare the data with presented literature data. These measurement protocols are the basis for the development of an open-source PCM database, which does not currently exist. 


\section{Materials and Methods}

\subsection{Materials}

The materials discussed and measured in this work are listed in Table 1. A variety of PCM classes have been investigated, including organic and inorganic PCM. Selection has been based on testing different PCM classes, including at least one per material class and melting point focusing on two aspects. First, temperatures relevant for building applications $\left(15^{\circ} \mathrm{C}, 30^{\circ} \mathrm{C}\right.$ and $\left.60^{\circ} \mathrm{C}\right)$, and second, covering the temperatures from $-50^{\circ} \mathrm{C}$ up to $190^{\circ} \mathrm{C}$ with several materials. Additionally, the criteria of commercial availability were taken into account. Furthermore, the measurement methodology is applied to both commercial PCM, as well as chemical substances.

Table 1. Materials investigated within this study and the corresponding purity, manufacturer and classification.

\begin{tabular}{|c|c|c|c|c|}
\hline PCM & CAS Number & Purity & Manufacturer & PCM Class \\
\hline 1-Octadecanol & $112-92-5$ & $99 \%$ & Sigma-Aldrich & Alcohol \\
\hline 1-Tetradecanol & $112-72-1$ & $97 \%$ & Sigma-Aldrich & Alcohol \\
\hline 1-Undecanol & $112-42-5$ & $99 \%$ & Sigma-Aldrich & Alcohol \\
\hline Ethyl octadecanoate & $111-61-5$ & $97 \%$ & Sigma-Aldrich & Ester \\
\hline Methyl hexadecanoate & $112-39-0$ & $99 \%$ & Sigma-Aldrich & Ester \\
\hline Methyl octadecanoate & $112-61-8$ & $96 \%$ & Sigma-Aldrich & Ester \\
\hline $\begin{array}{c}\left(\mathrm{MgCl}_{2}-\mathrm{Mg}\left(\mathrm{NO}_{3}\right)_{2}\right) \cdot 6 \mathrm{H}_{2} \mathrm{O} \\
(41 \mathrm{wt} \% / 59 \mathrm{wt} \%)\end{array}$ & $7786-30-3 / 13446-18-9$ & $98 \% / 99 \%$ & Sigma-Aldrich & Eutectic \\
\hline $\begin{array}{l}\text { Myristic acid-Stearic acid } \\
\quad(50 \mathrm{wt} \% / 50 \mathrm{wt} \%)\end{array}$ & $544-63-8 / 57-11-4$ & $99 \% / 98 \%$ & Sigma-Aldrich/Roth & Eutectic \\
\hline Hexadecanoic acid & $57-10-3$ & $99 \%$ & Sigma-Aldrich & Fatty acid \\
\hline Hexanoic acid & $142-62-1$ & $99 \%$ & Sigma-Aldrich & Fatty acid \\
\hline Methanoic acid & $64-18-6$ & $96 \%$ & Sigma-Aldrich & Fatty acid \\
\hline Octadecanoic acid & $57-11-4$ & $95 \%$ & Sigma-Aldrich & Fatty acid \\
\hline Propionic acid & 79-09-4 & $99.50 \%$ & Sigma-Aldrich & Fatty acid \\
\hline Crodatherm 17 & $\mathrm{n} / \mathrm{a}^{1}$ & As purchased ${ }^{1}$ & Croda Europe & Organic \\
\hline PEG 10000 & $25322-68-3$ & $\mathrm{n} / \mathrm{a}$ & Sigma-Aldrich & Organic \\
\hline Decane & $124-18-5$ & $99 \%$ & Sigma-Aldrich & Paraffin \\
\hline Dodecane & $112-40-3$ & $99 \%$ & Sigma-Aldrich & Paraffin \\
\hline Eicosane & $112-95-8$ & $99 \%$ & Sigma-Aldrich & Paraffin \\
\hline $\mathrm{CaBr}_{2} \cdot 6 \mathrm{H}_{2} \mathrm{O}$ & 71626-99-8 & $98 \%$ & Sigma-Aldrich & Salt hydrate \\
\hline $\mathrm{CaCl}_{2} \cdot 6 \mathrm{H}_{2} \mathrm{O}$ & 7774-34-7 & $98 \%$ & Sigma-Aldrich & Salt hydrate \\
\hline SAT & $\mathrm{n} / \mathrm{a}^{1}$ & As purchased ${ }^{1}$ & $\begin{array}{l}\text { Cowa Thermal } \\
\text { Solutions }\end{array}$ & Salt hydrate \\
\hline Dulcitol & $608-66-2$ & $99 \%$ & Sigma-Aldrich & Sugar alcohol \\
\hline meso-Erythritol & $149-32-6$ & $99 \%$ & Sigma-Aldrich & Sugar alcohol \\
\hline
\end{tabular}

${ }^{1}$ On commercial PCM, no data regarding CAS number, purity nor composition are given by the manufacturers. Any data not available is indicated by $n / a$.

\subsection{Equipment}

The investigated materials have either been tested as delivered, or, if necessary, were mixed and stirred at a temperature above their melting point for at least $30 \mathrm{~min}$. The following devices have been used for DSC measurements: The Mettler Toledo DSC 823e (Nänikon, Switzerland) with FRS 5+ sensor (Mettler Toledo, Nänikon, Switzerland) and TC100 Intracooler (Huber Kältemaschinenbau AG, Küsnacht, Switzerland), the Mettler Toledo DSC 3+ (Nänikon, Switzerland) with FRS 6+ sensors (Mettler Toledo, Nänikon, Switzerland) and both TC45/100 Intracoolers (Huber Kältemaschinenbau AG, Küsnacht, Switzerland). For the TGA measurements, a TGA/DSC 2, TGA/DSC 3+ and TGA/DSC 1 from Mettler Toledo all equipped with a DTA or DSC sensor were used. Equipment is shown in Supplementary Figure S1. 


\subsection{DSC Measurement Methodology}

\subsubsection{Preliminary Steps}

- The DSC was turned on approximately an hour before the measurement to allow the furnace to reach thermal equilibrium.

- Purge gas flow has been set to $20 \mathrm{~mL} \mathrm{~min}^{-1}$, and protective gas always $10 \mathrm{~mL} \mathrm{~min}^{-1}$ higher to avoid the entrance of sample vapors in the inner parts of the DSC. Nitrogen $\left(\mathrm{N}_{2}\right)$ has been used.

- The DSC has been checked regularly, at least once per month, and a calibration using Indium (In) prior every series of measurements was performed.

- Gloves and tweezers have been used for sample handling to avoid any contamination of the sample and the furnace.

\subsubsection{Sample Preparation}

A suitable sample crucible has been chosen considering compatibility with the measured PCM. Aluminum crucibles were mostly used, but if degradation or reaction of the PCM with the material of the crucible occurs, alumina crucibles could be considered.

When preparing the sample, a representative portion from the bulk material was chosen. If needed, it was mixed or stirred properly before preparing the sample.

If the sample was liquid, a syringe or a disposable pipette (made of glass or plastic) was used to pour the sample into the crucible. If the sample was solid, a micro spatula was used. The bottom of the pan was always fully covered when pouring the sample, to ensure proper heat transfer. Concerning amounts of substance in the crucible, $5 \mu \mathrm{L}$ were used when the sample is liquid and 5 to $10 \mathrm{mg}$ when solid. Three examples are shown in Supplementary Figure S2.

Both the mass of the empty pan and the poured sample have been measured using a scale with a maximum tolerance of $\pm 0.001 \mathrm{mg}$.

Any thermal alternation or contamination of the sample was avoided during sample preparation to avoid untrue results.

\subsubsection{Temperature Program}

The temperature program consists of several cycles at two distinct heating/cooling rates, as shown in Figure 1.

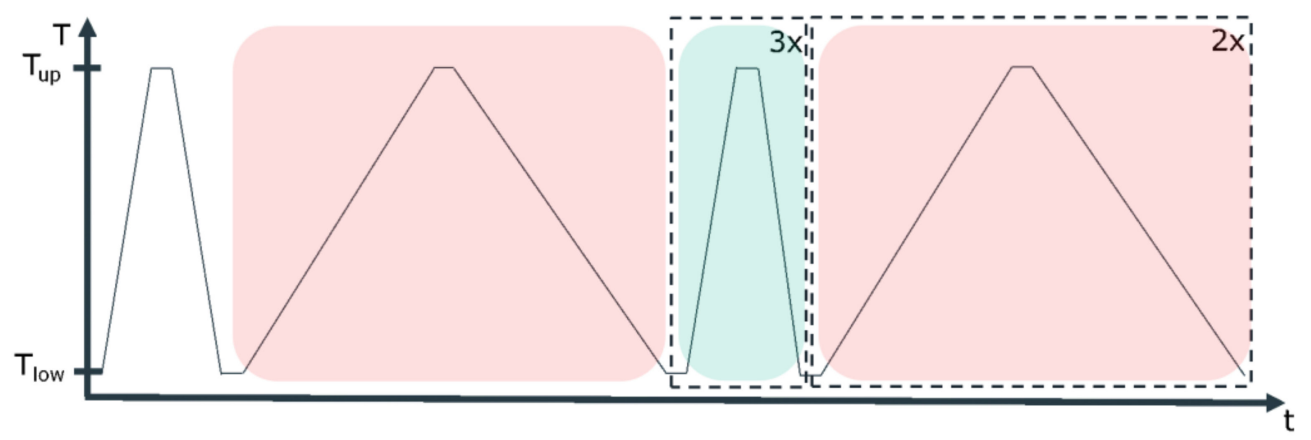

Figure 1. Complete temperature program for the differential scanning calorimetry (DSC) measurements. The upper and lower temperature limits, $\mathrm{T}_{\mathrm{up}}$ and $\mathrm{T}_{\mathrm{low}}$, are indicated, as well as the two heating rates applied in the experiment. The dashed boxes indicate the number of repetitions per heating rate, while the colored boxes indicate the segments used for analysis.

The faster heating rate is set to $10 \mathrm{~K} \mathrm{~min}^{-1}$, and the slower to $2 \mathrm{~K} \mathrm{~min}^{-1}$. The purpose of the first cycle is to delete the thermal history of the sample before the measurement. The other six cycles will be used to determine the phase change enthalpy and temperature, as will be explained later. 
The upper and lower limits, $\mathrm{T}_{\text {up }}$ and $\mathrm{T}_{\text {low }}$, are different for each PCM, as they are set with respect to its melting point, $\mathrm{T}_{\mathrm{m}}$, according to the following rule:

- $\quad \mathrm{T}_{\text {up }}=\mathrm{T}_{\mathrm{m}}+30 \mathrm{~K}$

- $\quad \mathrm{T}_{\text {low }}=\mathrm{T}_{\mathrm{m}}-20 \mathrm{~K}$

All the isothermal segments in between dynamic steps have a duration of 2 min to ensure thermal stabilization.

\subsubsection{Measurement}

In order to obtain a better statistical distribution of the results, at least 3 individual samples for each PCM were measured.

The crucibles have been checked by measuring the weight of the sample after the measurement to ascertain the sample is intact.

\subsubsection{Data analysis}

The last three segments at $10 \mathrm{~K} \mathrm{~min}^{-1}$ heating rate (indicated by green in Figure 1) are used to determine the phase change enthalpy, calculating the average and the standard deviation. The enthalpy is considered to be the area under the peak (see Figure 2), thus, the integral value of the differential heat flow in accordance with ASTM E 793 [20]. The use of the first derivative of the DSC curve was used to find more precisely both the start and the end of the melting, i.e. the points where the first derivative is zero for a certain length.

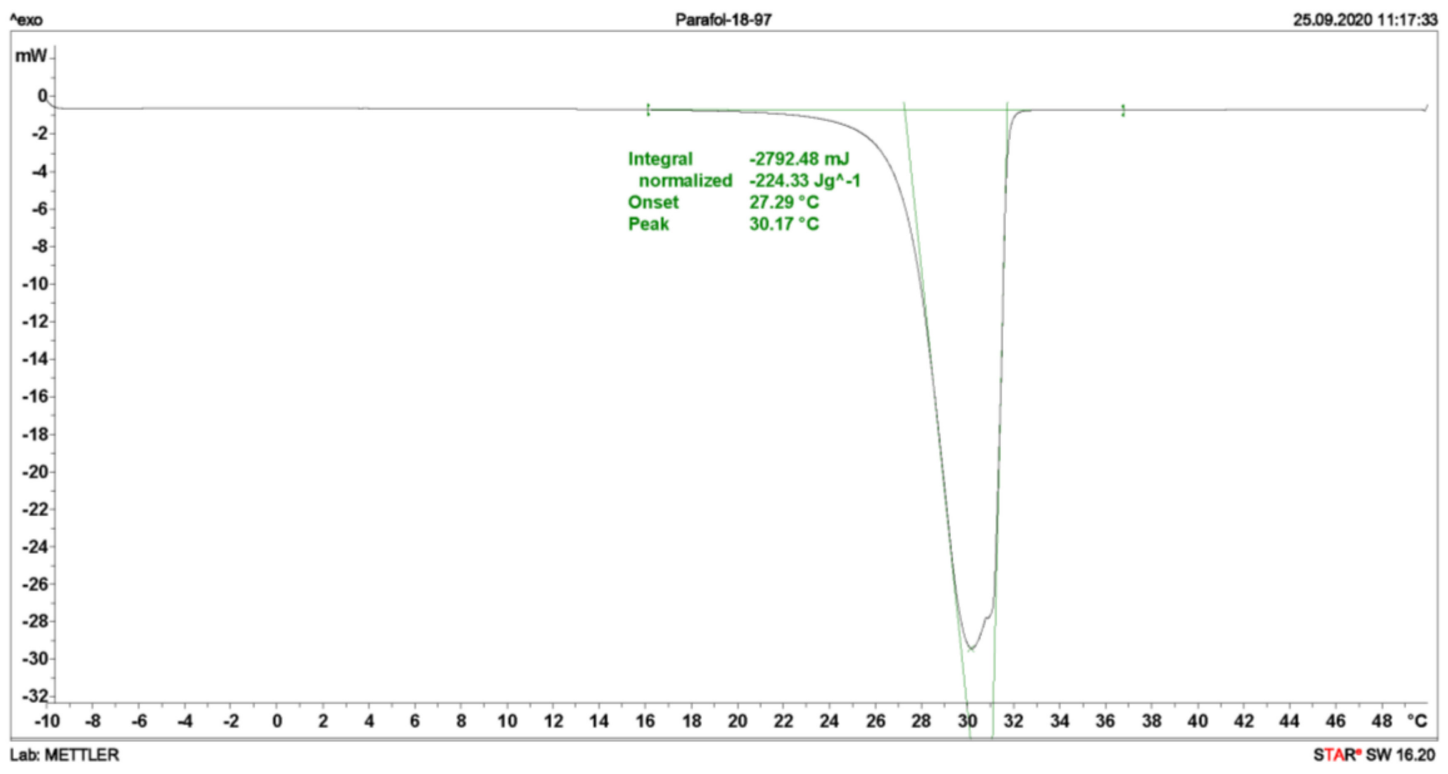

Figure 2. Graphical example of the determination of enthalpy, the integral of the signal with respect to the baseline before and after the peak, onset, as intersection between baseline and green tangential on the left, and peak, as highest point of the peak.

The three segments at $2 \mathrm{~K} \mathrm{~min}-1$ heating rate (indicated by red in Figure 1) have been used to determine the phase change temperature, calculating the average and the standard deviation. The onset temperature is considered to be the phase change temperature of the PCM, and it is obtained according to ASTM E 2253 [35]. Additionally, the peak temperature defined as maximum value per signal is also determined (see Figure 2).

The information provided by the cooling segments has been used to study the sub-cooling tendency of the PCM qualitatively. However, the DSC is not a suitable instrument to determine sub-cooling behavior for applied scale PCM. 


\subsection{TGA Measurement Methodology}

\subsubsection{Preliminary Steps}

The TGA was turned on the night prior to the measurement, such that the cryostat and the balance could stabilize properly overnight.

As long as the TGA is on, both protective and purge gas were allowed to flow through the device. Nitrogen $\left(\mathrm{N}_{2}\right)$ was used for common measurements. Oxygen $\left(\mathrm{O}_{2}\right)$ or air could be used as purge gas if oxidation or combustion of the sample are intended, but those measurements are not presented within this work. Protective gas flow was around $30 \mathrm{~mL} \mathrm{~min}^{-1}$, and purge gas flow always $10 \mathrm{~mL} \mathrm{~min}{ }^{-1}$ lower to avoid the entrance of vapors from the sample into the inner parts of the TGA.

The TGA has been checked regularly, at least once per month, and a valid calibration was performed at least once per year.

Tweezers and gloves have been used in every operation, as neither the crucibles nor the inner parts of the furnace should be touched with bare hands to avoid contamination of the sample or the furnace.

\subsubsection{Blank Measurement}

To correct the buoyancy effect, blank measurements were carried out before every set of measurements. An empty crucible was placed in the balance and a measurement launched with the same exact conditions (heating rate, gas flow, kind of crucible, etc.) as the ones expected to be used in the measurement of the sample.

If the TGA has not been used for a long period of time, three blank measurements have been performed. The first one was discarded and the most stable curve from the other two was chosen. A new blank measurement was launched every four samples (or every $5 \mathrm{~h}$ ) approximately, to ensure the use of a representative blank line. A new blank measurement was launched every time the conditions of the experiment changed (e.g. temperature range, gas flow or heating rate).

\subsubsection{Sample Preparation}

- The material of the crucible has to be compatible with the substances measured. Alumina crucibles have been used for all measurements.

- The sample should be representative of the bulk material, so it was stirred or mixed properly before extraction of the sample.

- A syringe or a disposable pipette was used when handling liquid samples. Solid samples were ground with a mortar, generating a powder as fine as possible. A micro spatula was then used to pour the sample into the crucible. The bottom of the crucibles should be fully covered. If grounding the PCM was not possible, samples with the same shape and size have been prepared to ensure repeatability.

- The amount of material poured on each crucible depends on the nature of the PCM: 5 to $10 \mathrm{mg}$ for organic PCM and 10 to $30 \mathrm{mg}$ for inorganic PCM, accounting for the general difference in density of these material categories, thus ensuring the coverage of the crucible bottom.

- The sample mass has been measured using a scale with a maximum tolerance of $\pm 0.001 \mathrm{mg}$. This mass has also been checked by the balance of the TGA itself.

- Caution was required to not alter the samples thermally before measurement, in order to avoid untrue results.

\subsubsection{Temperature Program}

When defining the temperature program of the experiment, the following steps are performed:

- An isothermal step of $10 \mathrm{~min}$ at $40{ }^{\circ} \mathrm{C}$, to ensure thermal stability of the sample before the heating step.

- A dynamic step from $40{ }^{\circ} \mathrm{C}$ to $600^{\circ} \mathrm{C}$ at a constant heating rate of $10 \mathrm{~K} \mathrm{~min}^{-1}$. 
- If a DSC/TGA has been used and the DSC curve is needed, an isothermal step of $10 \mathrm{~min}$ at $600{ }^{\circ} \mathrm{C}$ to correct the heat flow curve drift if necessary.

A diagram of the complete temperature program is represented in Figure 3.

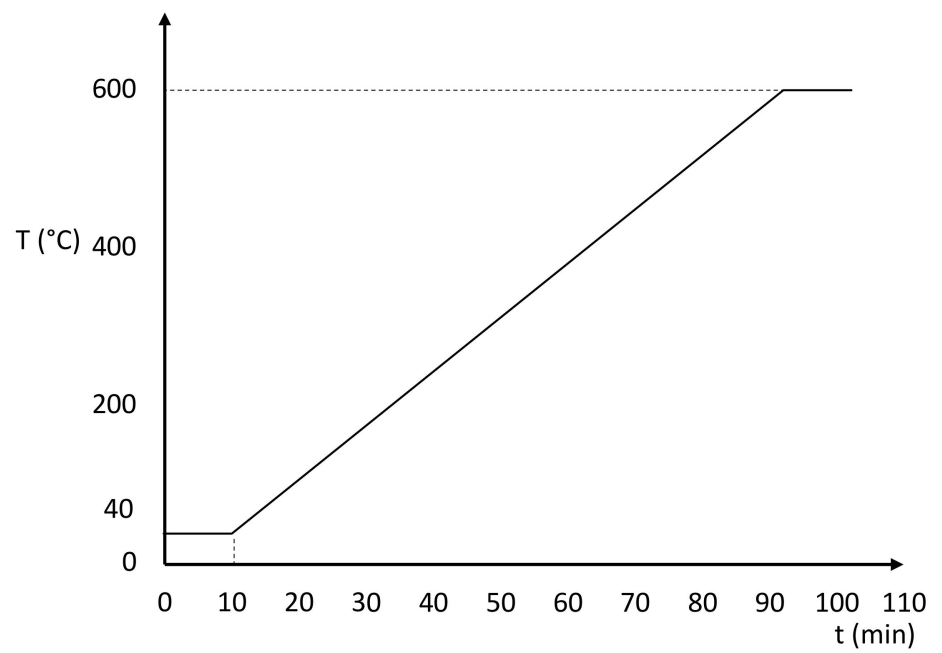

Figure 3. Complete temperature program for the thermogravimetric analysis (TGA) measurements. Duration and temperature of each step within the program are indicated, applied to all materials.

The upper limit of $600{ }^{\circ} \mathrm{C}$ has been found to be enough for the PCM measured within this work.

\subsubsection{Measurement}

Three different samples from each PCM have been prepared and measured, in order to obtain a better statistical distribution. If the alumina crucibles were reused, they were cleaned with a mixture $0.1 \mathrm{M}$ of hydrochloric acid $(\mathrm{HCl})$ in water, put in a muffle oven and kept at high temperatures for some time, to ensure that all the residues have been removed. When crucibles were too deteriorated, they were discarded.

\subsubsection{Data Analysis}

Before starting the analysis, the most stable baseline from the performed blank measurements was chosen and subtracted from the TGA curve of the measured PCM. To account for any thermal event reliably, the first derivative of the mass loss curve, DTG, was calculated.

The mass loss of a specific event was determined by tracing two horizontal lines over the TGA curve, one before the event and one after it, and taking the difference of these two horizontal values. The DTG curve was used to locate both the start and the end of the event, that is, the points in which the value of the curve is zero. This can be seen in Figure 4.

When several samples of the same material were compared, the relative mass loss curve has been used instead of the absolute one, to discard the effect of having different sample masses and being able to make a proper comparison.

If the TGA curve shows several steps, such as the TGA curve of a salt hydrate, each one of them has been analyzed as an independent event with its own onset temperature and mass loss.

We define the maximum application temperature of the PCM from the TGA experiments by considering the $2 \%$ onset temperature of thermal decomposition of the sample, calculated by evaluation of the first derivative curve (DTG), as shown in Figure 5. The 2\% threshold value is relative with respect to the peak height between the start temperature at $40^{\circ} \mathrm{C}$ and the first peak minimum on the DTG curve. The tangents of the evaluation and the horizontal line marking the $2 \%$ threshold are also shown in Figure 5. This methodology is based on analogue evaluations followed for other materials, 
such as in the definition of the glass transition temperature described in the standard DIN 65583 [36]. Following these steps, a quantitative analysis of the TGA curve was obtained.

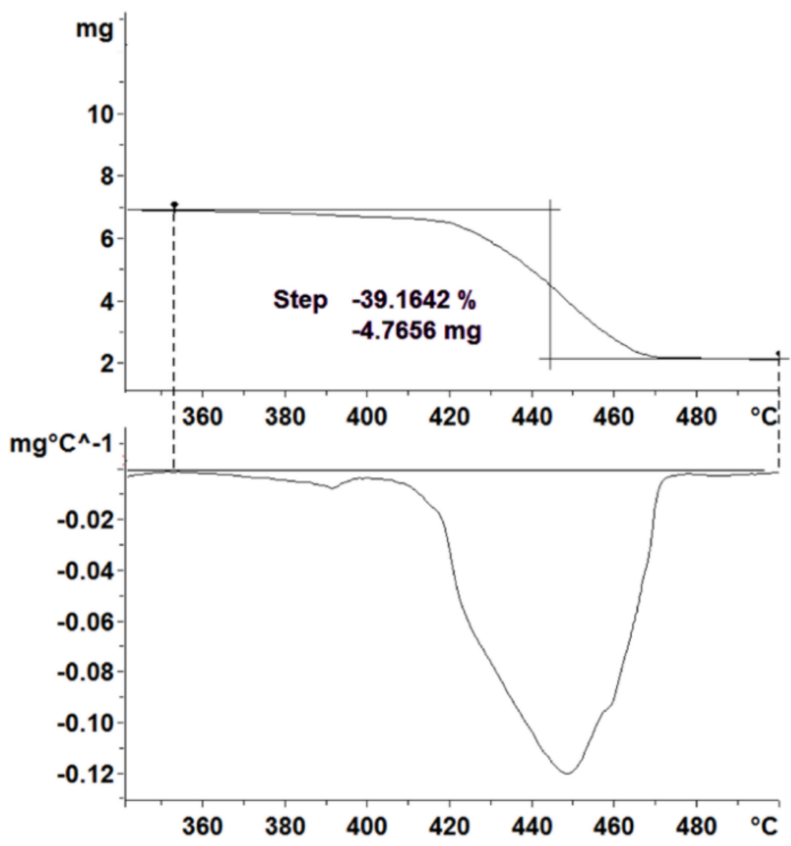

Figure 4. Calculation of the mass loss as relative change in mass of an event in a TGA curve. The beginning and end of a thermal event are determined using the first derivative curve (DTG) curve as shown in the lower graph.

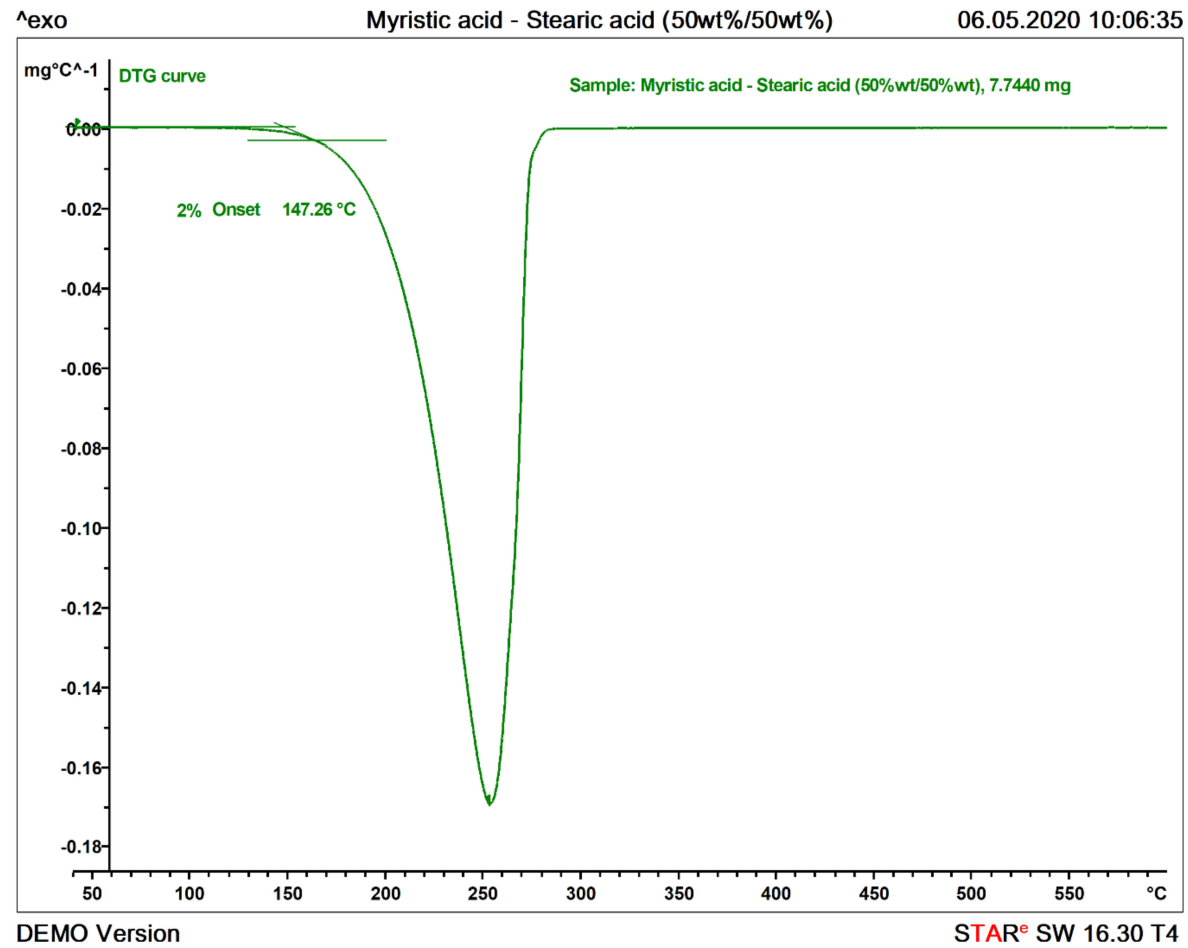

Figure 5. Calculation of the maximum operating temperature on a DTG curve. Maximum operating temperature is defined as the intersection of the tangential line on stable condition (left bound) and the tangential line at $2 \%$ change in mass loss rate (right bound). 


\section{Results and Discussion}

\subsection{DSC Measurements}

The following paragraphs will demonstrate the precision and accuracy of the method introduced in Section 2.3 for two different PCMs as an example. Furthermore, the extension to other PCM classes will be discussed on several examples from different classes each showing certain characteristics in the heat flux curve.

\subsubsection{Validation}

The proposed measurement methodology was validated using Parafol 18-97 (octadecane). This PCM has been measured and evaluated previously in a round robin experiment by Gschwander et al. [36]. The herein proposed measurement protocol has been repeated for 3 times, each indicated in Figure 6a by a different color. The measurements have shown that, throughout an experiment, the coefficient of variation $(\mathrm{CV})$ for enthalpy and onset temperature is between $0.02-0.12 \%$ and $0.06-0.38 \%$, respectively. This shows the high precision of the protocol. All those measured are shown in Supplementary Table S1.

In Figure $6 \mathrm{~b}$, the mean of each experiment is compared to the results of the round robin test performed by Gschwander et al. [37]. It is evident that the proposed protocol is as accurate as the methodology proposed by the aforementioned authors. The CVs for all measurements performed by them for enthalpy and onset temperature are $3.87 \%$ and $0.8 \%$, respectively. Including the results from our experiments, they become $3.71 \%$ and $0.75 \%$, indicating that the proposed methodology has at least the same accuracy.
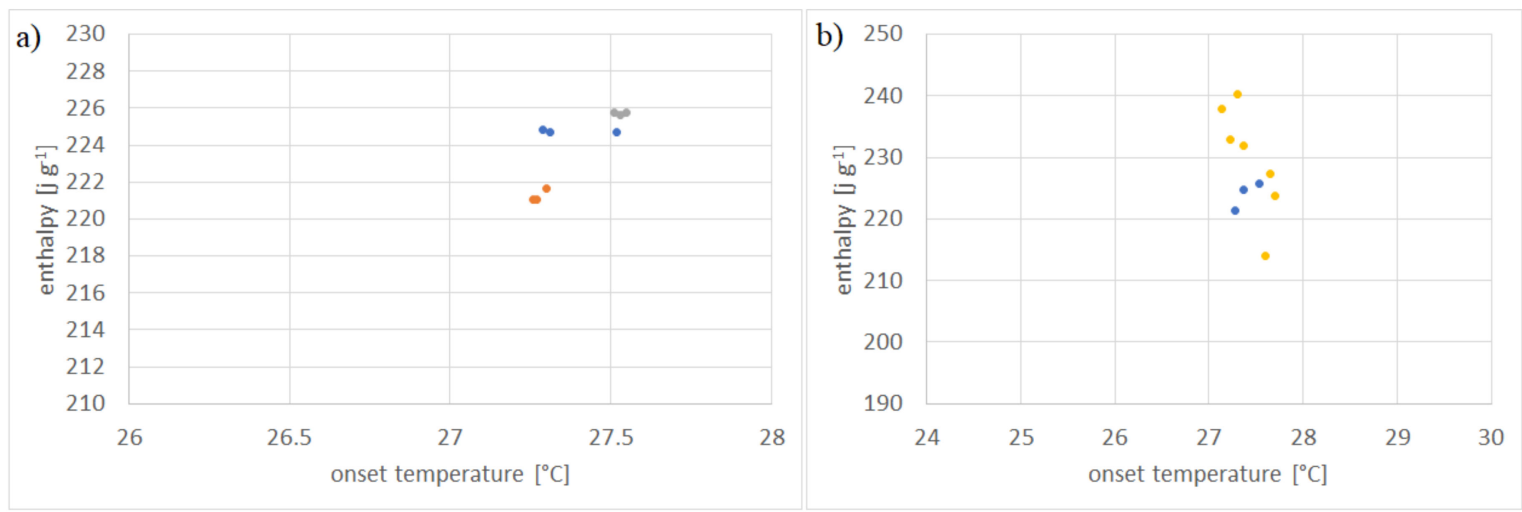

Figure 6. (a) Experimental results of enthalpy over onset temperature of Parafol 18-97 (octadecane) measured three times, as per measurement protocol suggested in Section 2.3. Each color depicts one measurement run. (b) Comparison of mean enthalpy and onset temperature from measurements (blue) with published data on standard DSC technique (yellow) [37].

In order to further validate the proposed measurement protocol, a small round robin test between the labs of Mettler Toledo and Hochschule Luzern and four different measurement devices (see Section 2.2) has been performed. The experiments were performed with Crodatherm 17 (see Table 2) from the same production lot and repeated between 4 and 8 times per device. A typical measurement curve and the results of the data analysis, as per Section 2.3.5, can be seen in Figure 7. Measured heat flux throughout the experiments is highly consistent, as indicated by the low CV values for enthalpy and onset temperature, being $0.41 \%$ and $0.25 \%$, respectively. Furthermore, the cooling curves are also shown as qualitative evaluation. Since Crodatherm17 is an organic PCM, it has low sub cooling, which is typical for this class of PCM $[9,38]$. 
Table 2. Comparability of method used by different operators and instruments for Crodatherm 17/DEV 2067. On each instrument, at least four and up to eight runs of the procedure described in Section 2.2 are considered. The mean \pm standard deviation and the coefficient of variation are given for each set of measurements.

\begin{tabular}{ccccccc}
\hline Laboratory & Operator & Instrument ${ }^{\mathbf{1}}$ & Enthalpy (J/g) & $\mathbf{C V ~ ( \% )}$ & $\begin{array}{c}\text { Onset } \\
\text { Temperature }\left({ }^{\circ} \mathbf{C}\right)\end{array}$ & CV (\%) \\
\hline HSLU $^{2}$ & 1 & $\mathrm{~A}$ & $165.32 \pm 0.63$ & 0.38 & $15.84 \pm 0.06$ & 0.37 \\
$\mathrm{HSLU}^{2}$ & 1 & $\mathrm{~B}$ & $156.41 \pm 0.72$ & 0.46 & $16.48 \pm 0.04$ & 0.26 \\
MT $^{3}$ & 2 & $\mathrm{C}$ & $171.15 \pm 0.61$ & 0.35 & $16.08 \pm 0.21$ & 1.3 \\
MT $^{3}$ & 2 & $\mathrm{D}$ & $169.32 \pm 0.71$ & 0.42 & $15.77 \pm 0.03$ & 0.19 \\
Mean & - & - & $165.55 \pm 6.56$ & 3.43 & $16.05 \pm 0.32$ & 1.73 \\
\hline
\end{tabular}

${ }^{1}$ A: Mettler Toledo DSC 823e, FRS 5+, TC100 Intracooler; B-D: Mettler Toledo DSC 3+, FRS 6+, TC45/100 Intracooler.

${ }^{2}$ Hochschule Luzern. ${ }^{3}$ Mettler Toledo.

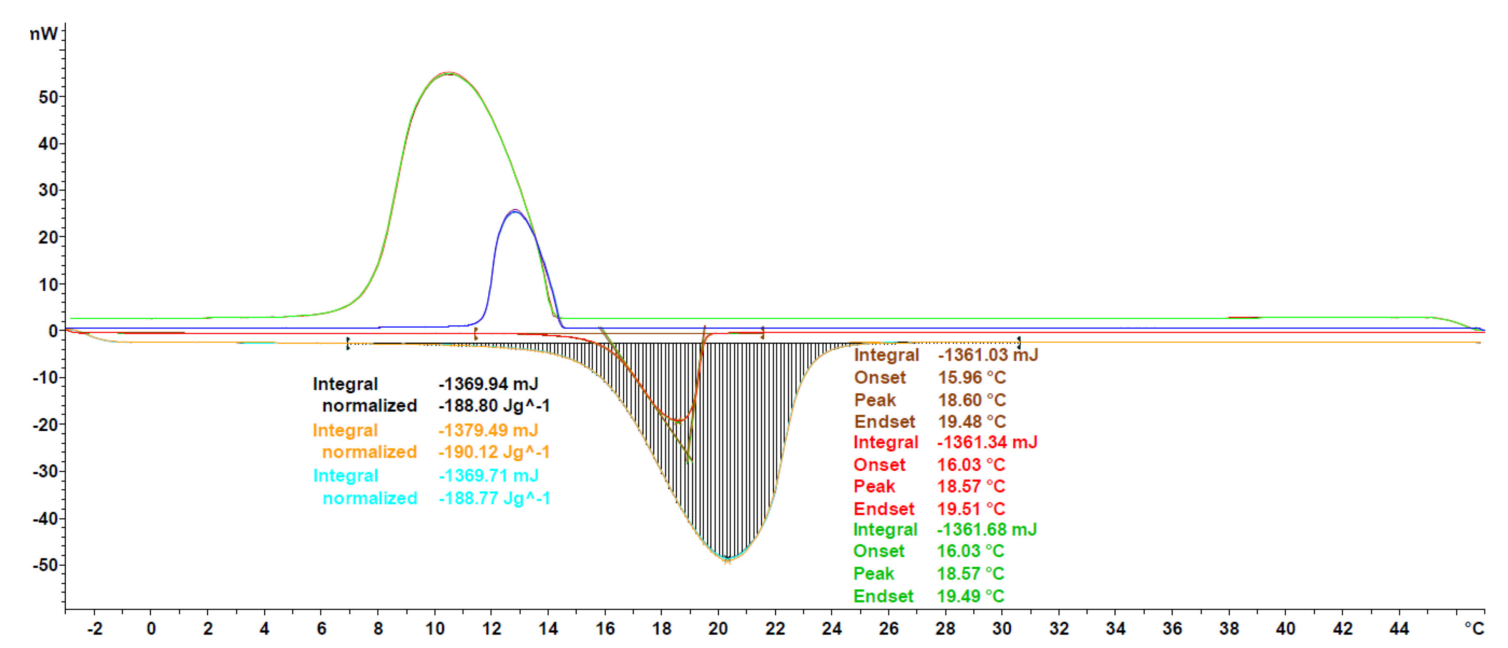

Figure 7. Heat flux curve of Crodatherm17 for a single experiment. Heating (lower) and cooling (upper) segments are shown, though only heating segments are evaluated for enthalpy and onset, peak and endset temperatures, as described in Section 2.3.5.

The mean results of each measurement series and the overall average are shown in Table 2. The calculated CV for enthalpy is in the same range as for the Parafol 18-97, indicating the robustness of the measurement protocol for other PCM classes. On the contrary, the CV for the onset temperature shows greater variance in different labs with a value of $1.73 \%$, which is almost double what has been reported for Parafol 18-97. Nevertheless, the value remains low and, having a closer look into mean and standard deviation, the measurement procedure can still be regarded as accurate.

To sum up the findings of the comparison between different methodologies and different laboratories, it should be pointed out that precision and accuracy are kept on a high quality level indicated by the small CV being $3.71 \%$ and $0.75 \%$, and thus smaller when including our results into the round robin results from [37]. Furthermore, the CV of measurements performed in different laboratories is in the same order of magnitude for enthalpy. Variations of the onset temperature are higher, but are regarded as small with respect to the absolute values.

An advantage of the methodology is the time needed to execute the measurement of a PCM. Compared to the RAL methodology [29], the DSC characterization presented in this work delivers results within approximately three days, while the RAL includes measurements of approximately seven days' duration. This leads to higher cost and thus to a lower number of PCM measured. 


\subsubsection{Extension to Different PCM Classes}

To show the suitability of the method for other PCM classes, a few example measurements are discussed in detail, along with its comparison with literature values and a description of typical effects encountered during measurements.

The first example is another organic PCM, octadecanoic acid, one of the most common saturated fatty acids [39]. Published values of enthalpy and melting temperature can vary largely, to an extent of $60 \mathrm{~J} \mathrm{~g}^{-1}$ and $18{ }^{\circ} \mathrm{C}$, respectively, as can be found on the National Institute of Standars and Technology (NIST) chemistry webbook, SRD 69 [40,41]. The values reported in this work are $230.81 \mathrm{~J} \mathrm{~g}^{-1}$ and $67.65{ }^{\circ} \mathrm{C}$, which is in good agreement with the majority of the NIST values, having a relative deviation of less than $5 \%$.

As can be seen in Figure 8, the generic melting/solidification behavior is similar to the ones of Crodatherm 17, though a difference in onset temperature for the cooling curves can be observed. This difference is an indicator of the validity of the classical nucleation theory and has been observed for a variety of other PCMs as well [42]. Due to the size-dependence through the number of possible nucleation sites, the effect of sub or even super cooling is more pronounced in DSC measurements, than it would be in applications. The temperature range of onset temperatures during solidification is given as a qualitative estimation of the tendency to supercool. Nevertheless, it must be mentioned here that the true amount of sub or super cooling has to be addressed and measured for each application in the corresponding sample size.

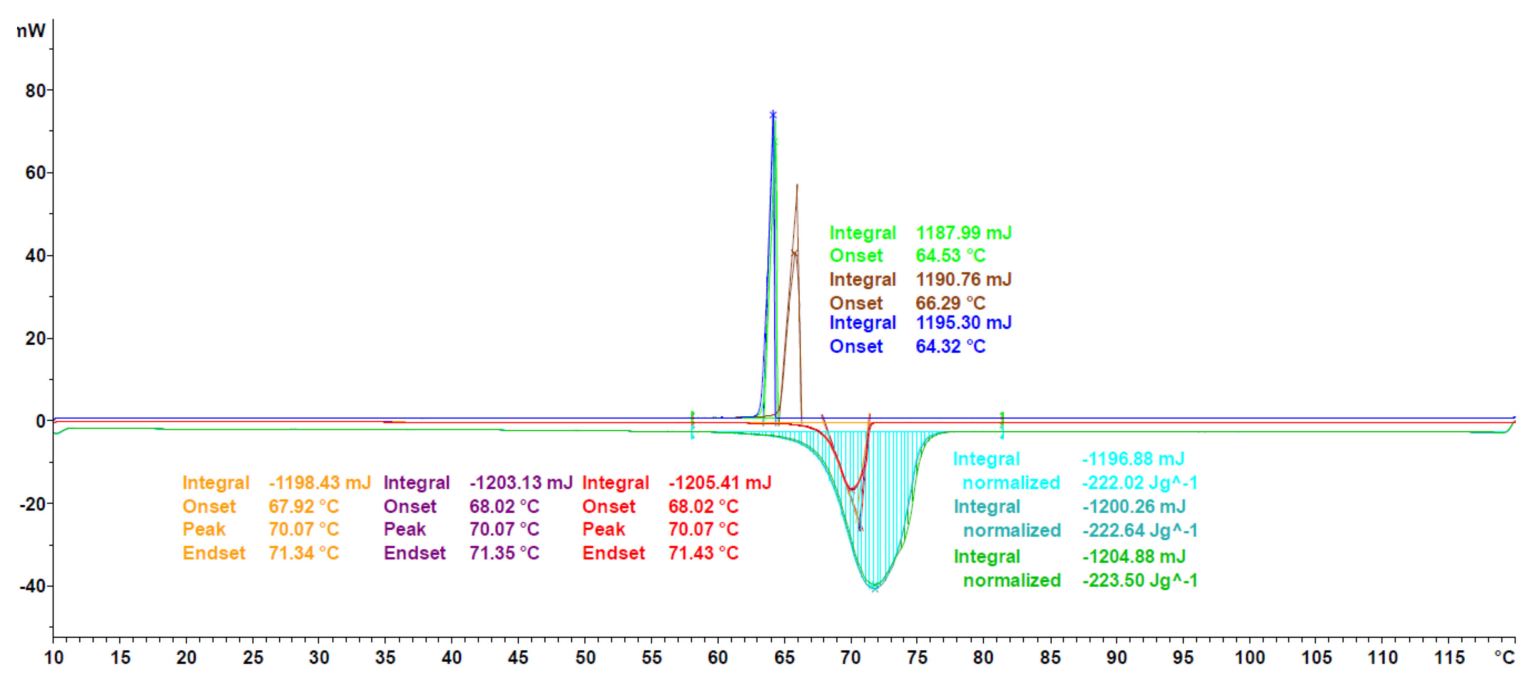

Figure 8. Heat flux curve of octadecanoic acid for a single experiment following the evaluation as per Section 2.3.5 is shown.

A further characteristic of organic PCM, polymorphism, can be seen for example in the measurement of 1-octadecanol, a fatty alcohol. As can be seen in Figure 9, two distinct peaks are visible in the cooling curves, indicating the formation of two different polymorphs. The confirmation for this observation is given through the existence of a slight shoulder on the left side of the measurement peaks of the heating curves. This phenomenon has already been described in literature by Ventolà et al. where also the thermo-physical properties are consistent with the ones measured in this work [43].

Additional thermo-physical data on 1-octadecanol can also be found in NIST chemistry webbook, SRD 69 [40]. The values stated for enthalpy have great variance, resulting in a CV of $30 \%$, while as the $\mathrm{CV}$ for melting temperature is at just $3 \%$. The results obtained in this work, $247.6 \mathrm{~J} \mathrm{~g}^{-1}$ and $56.61{ }^{\circ} \mathrm{C}$, are in good agreement with two of the four NIST stated enthalpy values, with a relative difference of less than $5 \%$, but between $0.6 \%$ and $8 \%$ off for stated temperatures. This offset could be due to the use of different experimental procedures, as results from other techniques apart from DSC are also 
published by NIST. Furthermore, it remains unclear if the onset temperature or the peak temperature has been selected as the phase change temperature.

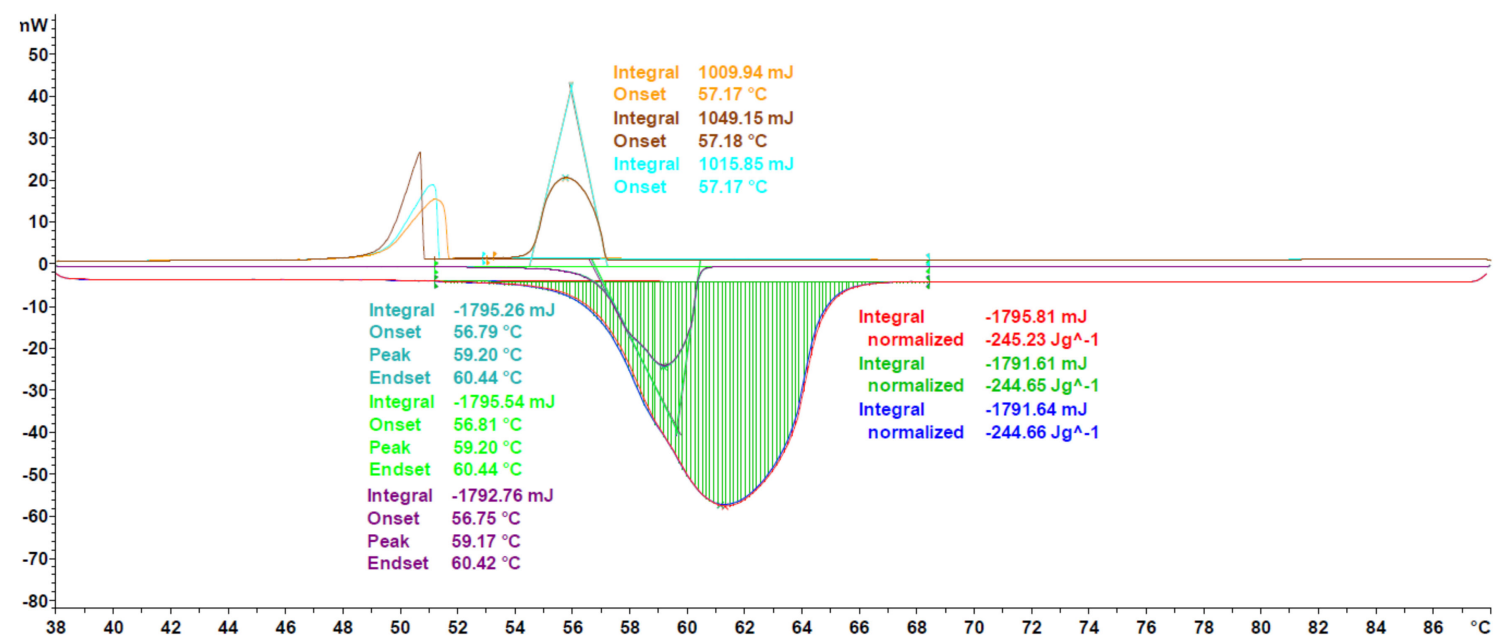

Figure 9. Heat flux curve of 1-octadecanol for a single experiment following the evaluation as per Section 2.3.5 is shown.

Calcium chloride hexahydrate, a salt hydrate, is shown in Figure 10, as an example of inorganic PCM. The main difference in the characteristic of inorganic PCM compared to organics is the highly pronounced super cooling effect, as has been described in literature [44]. Taking into account the mass dependence of the effect (as has already been mentioned), as well as the usually higher densities of salt hydrates (two to three times the one of organic PCM), the methodology has been adjusted to employ a greater mass (up to $40 \mathrm{mg}$ ) in the measurements of salt hydrates. Furthermore, the isothermal segments between cycles can be prolonged, in order to guarantee crystallization of the sample before the next heating segment.

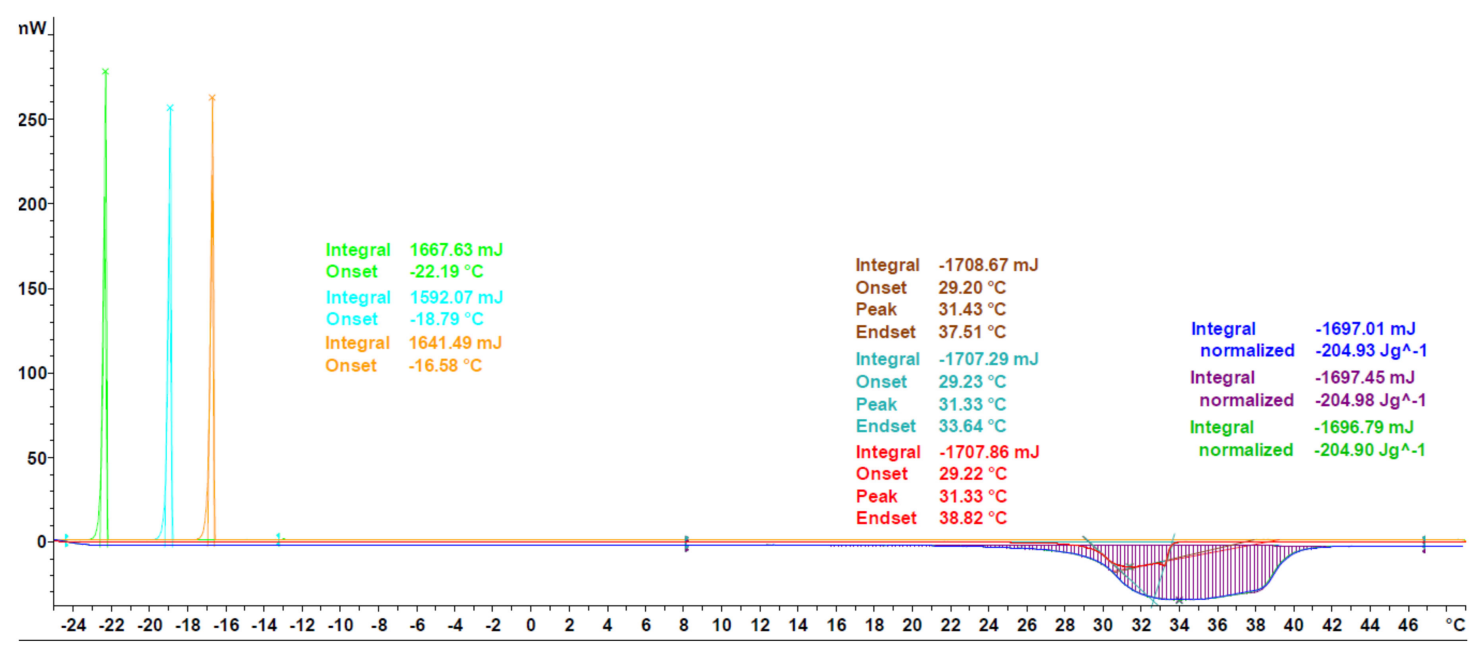

Figure 10. Heat flux curve of calcium chloride hexahydrate for a single experiment, following the evaluation as per Section 2.3.5, is shown.

Regarding the thermo-physical data, the value measured in this work is in good agreement with values reported by Schmit et al., for several manufactures, having a deviation of less than $3 \%$ for the mean value of enthalpy [45]. As shown in Figure 10, a single measurement can have a higher deviation, in the range of $5 \%$, but precision and accuracy can be optimized through the execution of at least three individual experiments with three evaluated segments. Furthermore, it has to be taken into 
account through the work of Schmit et al., a heating/cooling rate of $2 \mathrm{~K} \mathrm{~min}^{-1}$ is used to measure all thermo-physical data, whereas in this work, a heating rate of $10 \mathrm{~K} \mathrm{~min}^{-1}$ is used to determine enthalpy, which could lead to differences in the recorded values.

\subsection{TGA Measurements}

The proposed TGA methodology has also been validated through a series of measurements. The PCMs studied in this work have been measured in the same laboratories using a TGA 2 from Mettler Toledo.

Three samples of each PCM have been measured, and both the relative mass loss of each step observed in the TGA curve and the maximum operating temperature were extracted from the results, following the aforementioned definitions.

The relative mass loss of each step of some of the PCM has been studied, as well as their mean value, standard deviations and $\mathrm{CV}$, which are shown in Table 3 as an example. It shows that the coefficient of variation is low in all of them (lower than $1 \%$ ). This is a good indicator of the precision of the proposed measurement methodology.

Table 3. Relative mass loss in decomposition, mean, standard deviation and CV of some of the studied PCM.

\begin{tabular}{ccccc}
\hline PCM & Mass Loss (\%) & $\begin{array}{c}\text { Mean } \\
\mathbf{( \% )}\end{array}$ & $\begin{array}{c}\text { Standard Deviation } \\
\mathbf{( \% )}\end{array}$ & $\begin{array}{c}\text { CV } \\
\mathbf{( \% )}\end{array}$ \\
\hline \multirow{2}{*}{ 1-Tetradecanol } & 99.95 & & 0.02 & 0.02 \\
& 99.92 & 99.93 & & 0.35 \\
\hline Methyl & 99.92 & & 0.34 & \\
Hexadecanoate & 98.73 & 98.38 & & \\
\hline \multirow{2}{*}{ PEG 10.000 } & 98.04 & & 0.16 \\
& 98.39 & & & \\
\hline \multirow{2}{*}{ SAT } & 97.63 & 97.66 & & \\
& 97.84 & & & \\
\hline
\end{tabular}

Concerning the maximum operating temperature, the $2 \%$ onset temperature in the first derivative of the TGA curve criteria is proposed, due to similarities with the definition of the glass transition temperature in DMA experiments, where a $2 \%$ threshold in the curve is also specified. Further information can be found in the standard DIN 65583 [36]. The CV of the maximum operating temperature of the results has also been calculated, having a mean value of around $4 \%$ considering different PCM and devices, another good indicator of the quality of the procedure.

Examples of TGA and DTG curves with the extracted results are shown in Figure 11. Hexadecanoic acid experiments decompose in one single step, but two steps can be observed in the curve of calcium chloride hexahydrate, due to the dehydration. No lids were used with the crucibles in the measurements; so, in this case, water loss starts at a temperature below $40{ }^{\circ} \mathrm{C}$. This is the reason why no maximum operating temperature is displayed because, in this case, the proposed definition loses its meaning. 


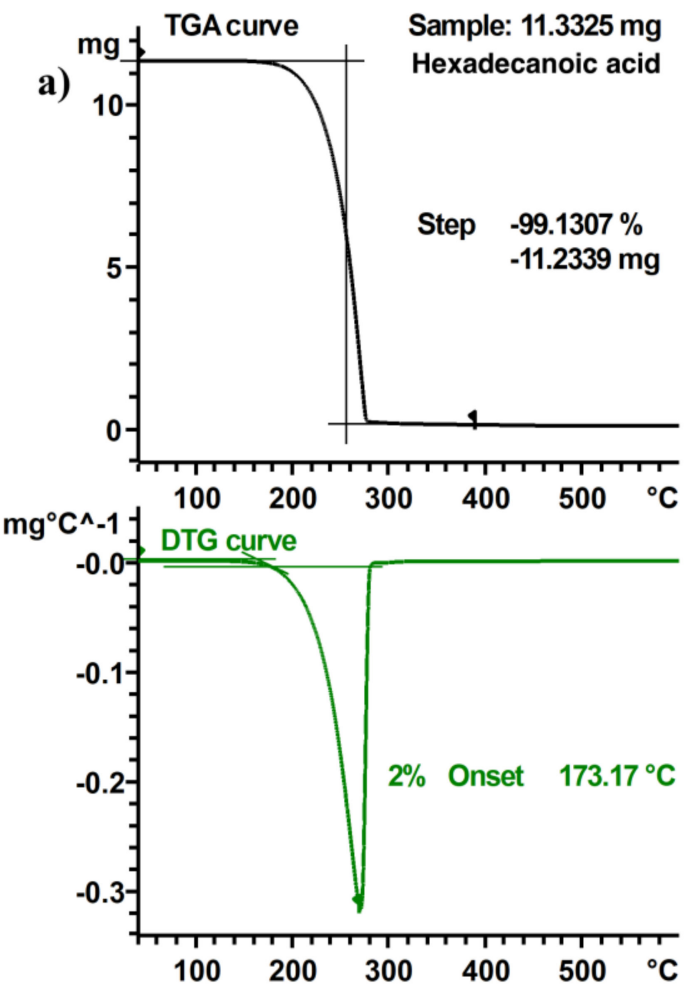

b)
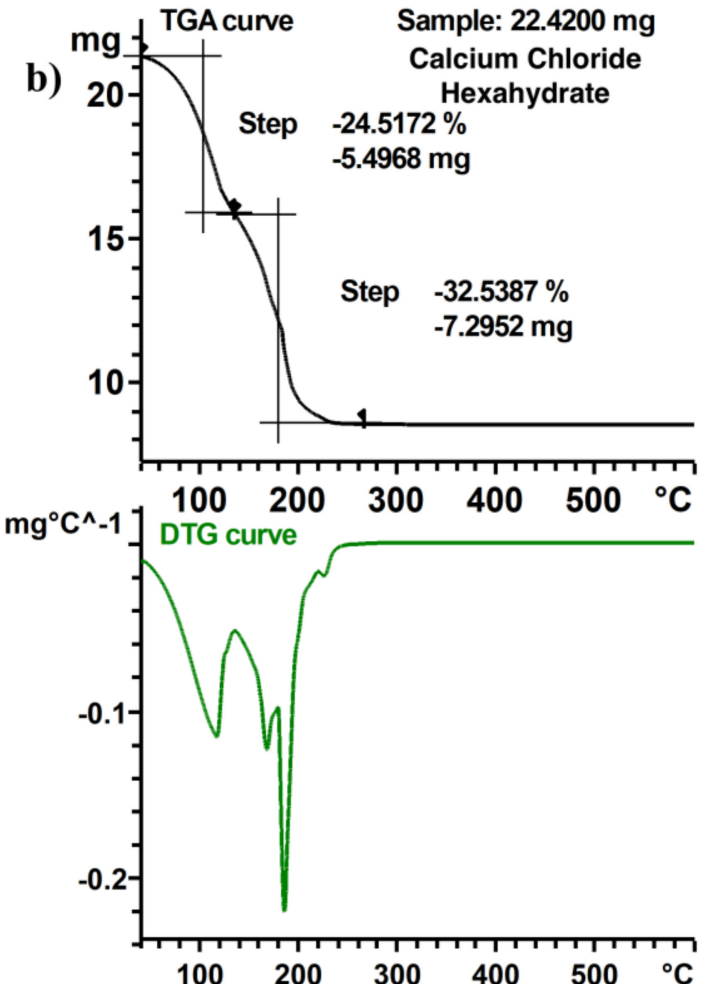

Figure 11. TGA and DTG curves of (a) hexadecanoic acid and (b) calcium chloride hexahydrate.

Comparison of the results with literature is difficult, as a new definition of maximum operating temperature is proposed. However, some other PCM have been measured to compare the experimental results with previously published data. The onset temperature and the temperature in which the mass loss rate is the maximum of three PCM have been compared with the results provided by several authors. The deviation of the results with each one of the papers is shown in Table 4 . It can be seen that, even for the same PCM and for the same property, different authors report different results. Furthermore, the same paper states that a high deviation for the onset temperature shows high deviation and a low deviation for the temperature of the maximum mass loss rate. This is most likely due to differences in the definitions of the measured properties. This qualitative comparison can only be considered as a proof of the need of a common methodology in the measurement of PCM properties.

Table 4. Mean values of the deviation of experimental results compared with the corresponding literature.

\begin{tabular}{cccc}
\hline Reference & PCM & $\begin{array}{c}\text { Deviation Onset } \\
\text { Temperature (\%) }\end{array}$ & $\begin{array}{c}\text { Deviation Temperature } \\
\text { Maxmium Mass } \\
\text { Loss Rate (\%) }\end{array}$ \\
\hline Xian Van et al. [46] & PEG 6000 & - & -3.36 \\
Zhi Chen et al. [47] & Stearic acid & 141.82 & 11.10 \\
Yaxue Lin et al. [48] & Stearic acid & 45.53 & 0.61 \\
Fang Tang et al. [25] & Octadecane & - & -15.62 \\
Hao Wang et al. [49] & Octadecane & 26.94 & - \\
Chaoen Li et al. [50] & Octadecane & 35.40 & - \\
\hline
\end{tabular}

In summary, it can be stated that measured mass loss curves show very small deviations per PCM, thus indicating the consistency of the methodology. The comparison with literature for TGA curves is rather difficult, due to the large deviations in methodology, and stresses the need for a precise methodology to allow for comparison in between PCM. 


\subsection{Database Measurements}

A total amount of 23 PCM from 9 different classes have been characterized through DSC/TGA, using the same measurement protocol as described in Section 2. An extract of the most important properties (i.e. melting enthalpy and onset, peak, crystallization onset and maximum operating temperature) and indication of standard deviation can be found in Table 5. Measuring such a large amount of different PCM is only possible due to the low cost of the methodologies presented, i.e. the smaller amount of time needed for measurements.

Table 5. Overview of the properties for all the PCM measured in this work. Given are the mean \pm standard deviation or the range of crystallization onset temperature as minimum and maximum measured interval.

\begin{tabular}{|c|c|c|c|c|c|c|c|}
\hline РCM & CAS & PCM Class & $\begin{array}{c}\text { Melting } \\
\text { Enthalpy (J/g) }\end{array}$ & $\begin{array}{l}\text { Melting } \\
\text { Onset }\left({ }^{\circ} \mathrm{C}\right)\end{array}$ & $\begin{array}{l}\text { Melting } \\
\text { Peak }\left({ }^{\circ} \mathrm{C}\right)\end{array}$ & $\begin{array}{l}\text { Crystallization } \\
\text { Onset }\left({ }^{\circ} \mathrm{C}\right)\end{array}$ & $\begin{array}{c}\text { Max. Operating } \\
\text { Temperature }\left({ }^{\circ} \mathrm{C}\right)\end{array}$ \\
\hline Methanoic acid & $64-18-6$ & Fatty Acid & $233.81 \pm 0.95$ & $-50.4 \pm 0.02$ & $-49.4 \pm 0.01$ & $-38.74--33.74$ & $\mathrm{n} / \mathrm{a}^{1}$ \\
\hline Decane & $124-18-5$ & Paraffin & $207.32 \pm 2.99$ & $-30.2 \pm 0.02$ & $-27.8 \pm 0.24$ & $-40.33--36.97$ & $\mathrm{n} / \mathrm{a}^{1}$ \\
\hline Dodecane & $112-40-3$ & Paraffin & $209.14 \pm 1.45$ & $-10.2 \pm 0.94$ & $-8 \pm 0.25$ & $-14.69--13.45$ & $\mathrm{n} / \mathrm{a}^{1}$ \\
\hline Hexanoic acid & $142-62-1$ & Fatty Acid & $141.21 \pm 0.58$ & $-4.7 \pm 0.2$ & $-1.2 \pm 0.06$ & $-14.59--12.09$ & $55.4 \pm 1.43$ \\
\hline 1-Undecanol & $112-42-5$ & Alcohol & $206.62 \pm 3.94$ & $14.5 \pm 0.27$ & $17.5 \pm 0.15$ & $10.35-11.54$ & $82.5 \pm 11.71$ \\
\hline $\mathrm{CaCl}_{2} \cdot 6 \mathrm{H}_{2} \mathrm{O}$ & $7774-34-7$ & Salt Hydrate & $197.97 \pm 6.22$ & $29.2 \pm 0.64$ & $31.7 \pm 0.73$ & $-24.1--16.4$ & $\mathrm{n} / \mathrm{a}^{1}$ \\
\hline Ethyl octadecanoate & $111-61-5$ & Ester & $188.74 \pm 1.49$ & $32.3 \pm 0.08$ & $34.5 \pm 0$ & $30.74-30.79$ & $149.4 \pm 17.28$ \\
\hline $\mathrm{CaBr}_{2} \cdot 6 \mathrm{H}_{2} \mathrm{O}$ & $71626-99-8$ & Salt Hydrate & $125.1 \pm 2.78$ & $32.7 \pm 0.08$ & $35.4 \pm 0.09$ & $23.37-24.46$ & $46.1 \pm 0.26$ \\
\hline Eicosane & $112-95-8$ & Paraffin & $246.44 \pm 5.02$ & $36 \pm 0.03$ & $37.8 \pm 0.06$ & $35.94-35.96$ & $154.5 \pm 6.88$ \\
\hline 1-Tetradecanol & $112-72-1$ & Alcohol & $226.26 \pm 2.47$ & $37.3 \pm 0.04$ & $39.7 \pm 0.1$ & $37.66-37.77$ & $110.8 \pm 5.15$ \\
\hline Methyl octadecanoate & $112-61-8$ & Ester & $229.16 \pm 4.26$ & $37.4 \pm 0.03$ & $40.1 \pm 0.06$ & $33.76-34.76$ & $141 \pm 4.06$ \\
\hline $\begin{array}{l}\text { Myristic acid-Stearic acid } \\
\qquad(50 w t \% / 50 w t \%)\end{array}$ & $\mathrm{n} / \mathrm{a}$ & $\begin{array}{l}\text { Organic } \\
\text { Eutectic }\end{array}$ & $179.97 \pm 6.1$ & $45.7 \pm 0.07$ & 0.12 & $43.58-43.96$ & $147.5 \pm 1.33$ \\
\hline PEG 10000 & $25322-68-3$ & Organic & $179.8 \pm 3.03$ & $59.3 \pm 0.3$ & $62.1 \pm 0.12$ & $46.52-50.14$ & $364.8 \pm 1.18$ \\
\hline Hexadecanoic acid & $57-10-3$ & Fatty Acid & $216.72 \pm 1.39$ & $62.1 \pm 0.01$ & $63.7 \pm 0.07$ & $59.55-60.65$ & $171.7 \pm 2.68$ \\
\hline Octadecanoic acid & $57-11-4$ & Fatty Acid & $228.26 \pm 1.01$ & $67.8 \pm 0.2$ & $70 \pm 0.1$ & $63.66-66.31$ & $195.4 \pm 1.4$ \\
\hline meso-Erythritol & $149-32-6$ & Sugar Alcohol & $337.76 \pm 7.51$ & $118.7 \pm 0.21$ & $120.4 \pm 0.17$ & $22.99-32.29$ & $192.3 \pm 1.84$ \\
\hline Dulcitol & $608-66-2$ & Sugar Alcohol & $352.91 \pm 8.18$ & $186.6 \pm 0.51$ & $189 \pm 0.31$ & $105.35-119.2$ & $242.4 \pm 1.57$ \\
\hline
\end{tabular}

1: n/a: not applicable.

The PCMs have been selected using several criteria. Firstly, a manifold of 9 different PCM classes was selected to enable a quantitative comparison between classes for any specific application. Furthermore, most applications require a specific temperature range from the storage material, so PCMs with similar meting/crystallization temperatures were selected. For example, PCMs with phase change temperatures around $30^{\circ} \mathrm{C}$ or $60^{\circ} \mathrm{C}$ are of importance in the building sector, namely residential heating and domestic hot water [51].

Additional PCMs were chosen to show the versatility of the method over a wide temperature range; from -50 to $186^{\circ} \mathrm{C}$. Only some TGA measurements revealed a loss of material within the isothermal segment at the beginning, which indicates a maximum operating temperature below $40^{\circ} \mathrm{C}$. Within Table 5, PCMs exhibiting such a mass loss are indicated with "not applicable" (n/a), since an evaluation with the methodology proposed is not meaningful.

Figure 12 shows the two most important properties, enthalpy over onset temperature for the PCM given in Table 5. One can see that that the values for enthalpy are distributed in between 120 and $250 \mathrm{~J} \mathrm{~g}^{-1}$. The consistency of the methodology allows for comparison and correlation between different PCMs, and even between PCM classes. It has to be mentioned though, that the number of data points is too small to draw any conclusions about the nature of the distribution, but there seems to be a uniform distribution over all temperatures below $100{ }^{\circ} \mathrm{C}$. It would require a systematic analysis per class, such as for example, the one conducted by Ravotti et al. [9]. The only PCMs measured above $100{ }^{\circ} \mathrm{C}$ show substantially higher enthalpy, with values around $350 \mathrm{~J} \mathrm{~g}^{-1}$. Both of them are sugar alcohols which are known to have higher enthalpy values than other PCM, but are only available with melting points 
around $100^{\circ} \mathrm{C}$ and above [52-54]. These properties correspond well with what is reported in literature for PCM classes [55].

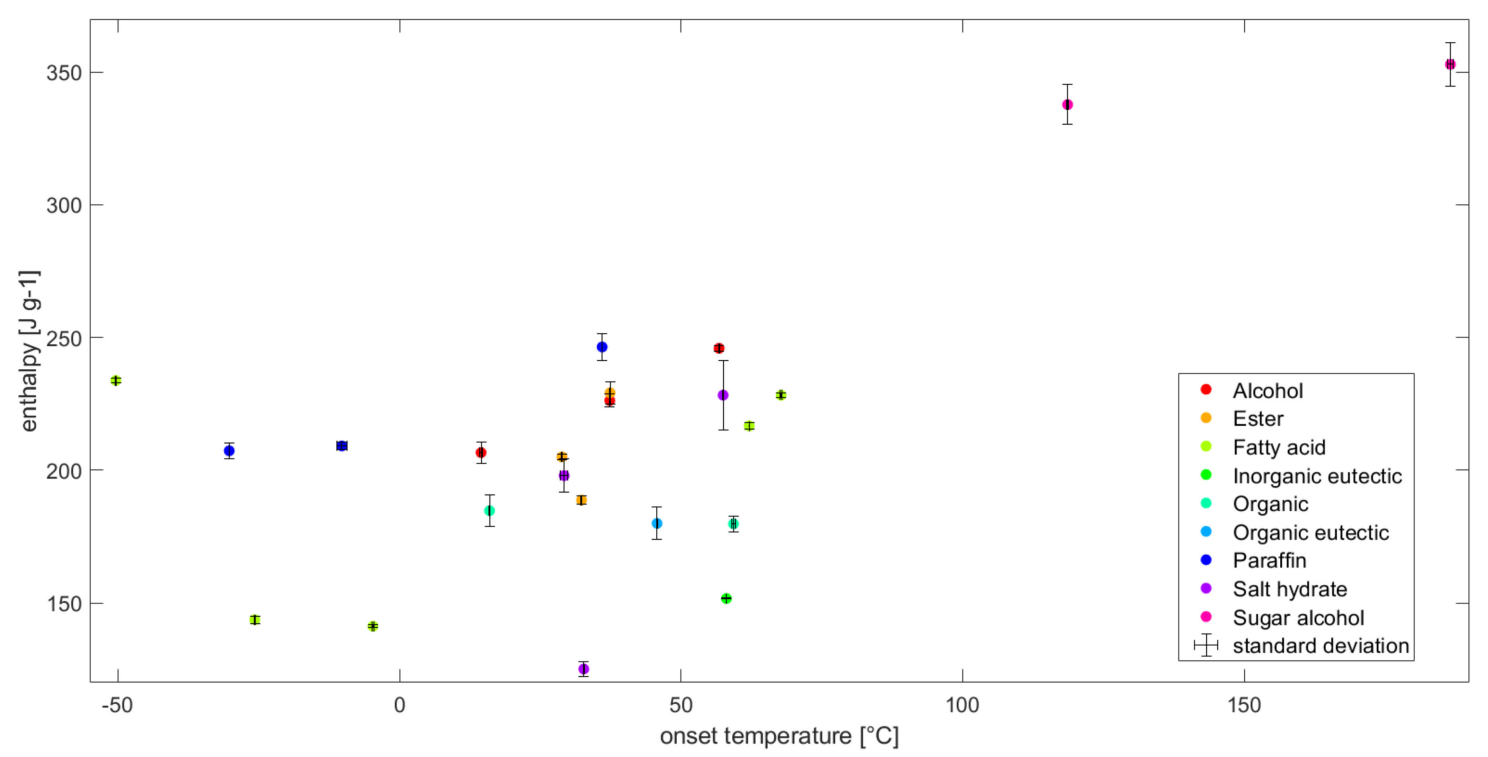

Figure 12. Enthalpy over onset temperature of materials, as given in Table 5. Additionally, the standard deviation is indicated by error bars showing their narrow distribution around the mean.

\section{Conclusions and Outlook}

It is shown in this work that a consistent DSC and TGA methodology can be used to consistently measure and compare thermo-physical PCM properties. This facilitates the selection process for a specific application, and guarantees true conclusions, which could not necessarily be drawn from a literature review, due to the reported differences in measurement protocols. Furthermore, a new definition of maximum operating temperature has been introduced, which accounts for first changes in mass upon temperature exposure, and could indicate irreversible degradation processes, regardless of PCM class.

The choice of two different heating/cooling rates became obvious to guarantee precision and accuracy for the two most relevant thermo-physical properties, enthalpy and onset temperature. Enthalpy measurement has better accuracy for higher heating/cooling rates, while the higher heating rate significantly influences the onset temperature. Thus, a second heating/cooling rate was introduced in order to assess the onset temperature in the same experiment. Additionally, the repetition of cycles allows for having statistics within one experiment and additionally shows consistency of phase change, which is essential for almost all applications.

When extending the measurement procedure to different PCM classes, it became apparent that minor changes have to be introduced for certain materials. For the DSC protocol, it was shown that materials with a super cooling tendency need an adjustment in order to observe crystallization events and thus subsequent melting of the PCM, in order to be able to evaluate the thermo-physical properties. The adjustments consist of decreasing the lower temperature limit, increasing the time of isothermal segments in between cycles and allowing for higher sample masses. The first two adjustments do not significantly influence the evaluation, since the heating/cooling rates are kept constant. The weight adjustment can have a minor influence on both properties, but is needed to create crystallization events. Furthermore, it must be mentioned that an increase in mass is generally allowed for PCM with higher densities, thus volume is kept similar.

The presented methodology has some limitations, which include super cooling, size, starting temperature and encapsulation, leading to a possible lack of measured values. The super cooling limits the application of the DSC methodology, such that a stable super cooling will not lead to 
recrystallization and thus no detection of further melting events throughout the procedure. This could be partially omitted by increasing the holding times at the lower temperature. Limitation of size for DSC methodology has been introduced and further enhances super cooling tendency. Therefore, caution has to be taken by reporting the super-cooling temperature, which can largely deviate from application specific size of sample. A large limitation of the TGA methodology is the starting temperature at $40^{\circ} \mathrm{C}$ to reach thermal equilibrium, which is given through the instrument used. This limitation leads to unavailable data for PCM, where evaporation starts within the isothermal segment. Lastly, it needs to be stated that the results obtained are for unmodified PCM, except commercially available ones measured, and thus, the results do not include any change in properties through addition of nucleators, thickeners or any kind of encapsulation.

It is worth mentioning that the data published in this work will be included in an open-source PCM database and into a so-called reference library of the StarE software.

In conclusion, the measurement procedure presented in this work can be successfully applied to a manifold of PCM classes delivering a concise set of thermo-physical properties. This is the basis to develop an open-source PCM database with comparable and reliable data, in order to facilitate the market penetration of PCM based products.

Further research will be directed to include further important properties for a thorough selection process, such as specific heat capacity, thermal conductivity and density. The corresponding measurement methodologies require standardization, and thus comparative studies with appropriate design. Additionally, the extension of the presented methodology to encapsulated PCM could be addressed and validated. At last, the measurement of further PCM with the presented methodology is regarded to be the most important point, in order to enable correlations and support a sound material selection. A strong emphasis on the inclusion of materials not yet characterized is suggested.

Supplementary Materials: The following are available online at http://www.mdpi.com/1996-1944/13/20/4486/s1, Figure S1: a) DSC 3+, b) DSC 823e, c) TGA/DSC 2, Figure S2: Three examples of prepared material for DSC measurement, Table S1: Enthalpy and onset temperature measured for Parafol 18-97 (octadecane) including their mean and standard deviation.

Author Contributions: L.M. carried out the conceptualization; L.M., G.R.-P. and A.B. developed and validated the methodology and performed the investigations; L.M. and A.B. curated the data; L.M., G.R.-P. and A.B. wrote and prepared the original draft ; N.M.-R., F.A. and J.W. reviewed and edited the paper and supervised the project; L.M., G.R.-P. and A.B. checked the visualization; L.M. administrated the project; N.M.-R., F.A. and J.W. were in charge of the funding acquisition. All authors have read and agreed to the published version of the manuscript.

Funding: This work is part of the Doctoral Thesis of Gabriel Rubio Pérez, and it has been partially funded by the Ministerio de Cienca, Innovación y Universidades (Spain).

Acknowledgments: The authors would like to thank Urs Jörimann from Mettler Toledo Material Characterization group for the generous support by providing additional instruments (microscale, TGA, automatisation equipment) and measurement resources at their facilities.

Conflicts of Interest: The authors declare no conflict of interest.

\section{References}

1. Grosspietsch, D.; Saenger, M.; Girod, B. Matching decentralized energy production and local consumption: A review of renewable energy systems with conversion and storage technologies. Wires Energy Environ. 2019, 8, e336. [CrossRef]

2. Isaac, M.; van Vuuren, D.P. Modeling global residential sector energy demand for heating and air conditioning in the context of climate change. Energy Policy 2009, 37, 507-521. [CrossRef]

3. Nazir, H.; Batool, M.; Bolivar Osorio, F.J.; Isaza-Ruiz, M.; Xu, X.; Vignarooban, K.; Phelan, P.; Inamuddin Kannan, A.M. Recent developments in phase change materials for energy storage applications: A review. Int. J. Heat Mass Transf. 2019, 129, 491-523. [CrossRef]

4. Palacios, A.; de Gracia, A.; Cabeza, L.F.; Julià, E.; Fernández, A.I.; Barreneche, C. New formulation and characterization of enhanced bulk-organic phase change materials. Energy Build. 2018, 167, 38-48. [CrossRef] 
5. Eanest Jebasingh, B.; Valan Arasu, A. A comprehensive review on latent heat and thermal conductivity of nanoparticle dispersed phase change material for low-temperature applications. Energy Storage Mater. 2020, 24, 52-74. [CrossRef]

6. Martín, M.; Villalba, A.; Inés Fernández, A.; Barreneche, C. Development of new nano-enhanced phase change materials (NEPCM) to improve energy efficiency in buildings: Lab-scale characterization. Energy Build. 2019, 192, 75-83. [CrossRef]

7. Oh, K.; Kwon, S.; Xu, W.; Wang, X.; Toivakka, M. Effect of micro- and nanofibrillated cellulose on the phase stability of sodium sulfate decahydrate based phase change material. Cellulose 2020, 27, 5003-5016. [CrossRef]

8. Kumar, N.; Ness, R.V.O.N.; Chavez, R., Jr.; Banerjee, D.; Muley, A.; Stoia, M. Experimental Analysis of Salt Hydrate Latent Heat Thermal Energy Storage System With Porous Aluminum Fabric and Salt Hydrate as Phase Change Material With Enhanced Stability and Supercooling. J. Energy Resour. Technol. 2020, 143. [CrossRef]

9. Ravotti, R.; Lardon, N.; Stamatiou, A.; Worlitschek, J.; Fischer, L.; Fellmann, O. Analysis of Bio-Based Fatty Esters PCM's Thermal Properties and Investigation of Trends in Relation to Chemical Structures. Appl. Sci. 2019, 9, 225. [CrossRef]

10. Ravotti, R.; Fellmann, O.; Lardon, N.; Fischer, L.J.; Stamatiou, A.; Worlitschek, J. Investigation of Lactones as Innovative Bio-sourced PCM for Latent Heat Storage. Molecules 2019, 24, 1300. [CrossRef]

11. Fabiani, C.; Pisello, A.L.; Barbanera, M.; Cabeza, L.F. Palm oil-based bio-PCM for energy efficient building applications: Multipurpose thermal investigation and life cycle assessment. J. Energy Storage 2020, 28, 101129. [CrossRef]

12. Faraj, K.; Khaled, M.; Faraj, J.; Hachem, F.; Castelain, C. Phase change material thermal energy storage systems for cooling applications in buildings: A review. Renew. Sustain. Energy Rev. 2020, 119, 109579. [CrossRef]

13. Prajapati, D.G.; Kandasubramanian, B. A Review on Polymeric-Based Phase Change Material for Thermo-Regulating Fabric Application. Polym. Rev. 2020, 60, 389-419. [CrossRef]

14. Barreneche, C.; Navarro, M.E.; Cabeza, L.F.; Fernández, A.I. New database to select phase change materials: Chemical nature, properties, and applications. J. Energy Storage 2015, 3, 18-24. [CrossRef]

15. Barreneche, C.; Navarro, H.; Serrano, S.; Cabeza, L.F.; Fernández, A.I. New Database on Phase Change Materials for Thermal Energy Storage in Buildings to Help PCM Selection. Energy Procedia 2014, 57, 2408-2415. [CrossRef]

16. Lager, D. Evaluation of Thermophysical Properties for Thermal Energy Storage Materials-Determining Factors, Prospects and Limitations; Technische Universität: Wien, Austria, 2018.

17. Barreneche, C.; Pisello, A.L.; Fernández, A.I.; Cabeza, L.F. Experimental Methods for the Characterization of Materials for Latent Thermal Energy Storage. In Recent Advancements in Materials and Systems for Thermal Energy Storage; Frazzica, A., Cabeza, L.F., Eds.; Springer: Berlin/Heidelberg, Germany, 2019; pp. 89-101. ISBN 978-3-319-96639-7.

18. DIN 53765. Testing of Plastics and Elastomeres; Thermal Analysis; DSC-Method; Deutsches Institut fur Normung E.V. (DIN): Berlin, Germany, 1994.

19. ISO 11357-3. Plastics-Differential Scanning Calorimetry (DSC)-Part 3: Determination of Temperature and Enthalpy of Melting and Crystallization; International Organization for Standardization (ISO): Geneva, Switzerland, 2011.

20. ASTM E793. Standard Test Method for Enthalpies of Fusion and Crystallization by Differential Scanning Calorimetry; ASTM International (ASTM): West Conshohocken, PA, USA, 2012. [CrossRef]

21. ASTM D4318. Standard Test Method for Transition Temperatures and Enthalpies of Fusion and Crystallization of Polymers by Differential Scanning; ASTM International (ASTM): West Conshohocken, PA, USA, 2012. [CrossRef]

22. Haillot, D.; Bauer, T.; Kröner, U.; Tamme, R. Thermal analysis of phase change materials in the temperature range $120-150{ }^{\circ} \mathrm{C}$. Acta $\mathbf{2 0 1 1}, 513,49-59$. [CrossRef]

23. Harish, S.; Orejon, D.; Takata, Y.; Kohno, M. Thermal conductivity enhancement of lauric acid phase change nanocomposite with graphene nanoplatelets. Appl. Eng. 2015, 80, 205-211. [CrossRef]

24. Pielichowski, K.; Flejtuch, K. Differential scanning calorimetry studies on poly(ethylene glycol) with different molecular weights for thermal energy storage materials. Polym. Adv. Technol. 2002, 13, 690-696. [CrossRef] 
25. Tang, F.; Cao, L.; Fang, G. Preparation and thermal properties of stearic acid/titanium dioxide composites as shape-stabilized phase change materials for building thermal energy storage. Energy Build. 2014, 80, 352-357. [CrossRef]

26. Tong, B.; Tan, Z.C.; Shi, Q.; Li, Y.S.; Yue, D.T.; Wang, S.X. Thermodynamic investigation of several natural polyols (I): Heat capacities and thermodynamic properties of xylitol. Acta 2007, 457, 20-26. [CrossRef]

27. Barreneche, C.; Solé, A.; Miró, L.; Martorell, I.; Fernández, A.I.; Cabeza, L.F. Study on differential scanning calorimetry analysis with two operation modes and organic and inorganic phase change material (PCM). Acta 2013, 553, 23-26. [CrossRef]

28. Castellón, C.; Günther, E.; Mehling, H.; Hiebler, S.; Cabeza, L.F. Determination of the enthalpy of PCM as a function of temperature using a heat-flux DSC-A study of different measurement procedures and their accuracy. Int. J. Energy Res. 2008, 32, 1258-1265. [CrossRef]

29. RAL Institute. Quality $\mathcal{E}$ Testing Specifications for Phase Change Materials; RAL Deutsches Institut für Gütesicherung und Kennzeichnung e.V.: Bonn, Germany, 2018.

30. Gschwander, S.; Lazaro, A.; Cabeza, L.F.; Günther, E.; Fois, M.; Chui, J. Development of a Test-Standard for PCM and TCM Characterization Part 1: Characterization of Phase Change Materials; International Energy Agency (IEA): Paris, France, 2011.

31. Lazaro, A.; Peñalosa, C.; Solé, A.; Diarce, G.; Haussmann, T.; Fois, M.; Zalba, B.; Gshwander, S.; Cabeza, L.F. Intercomparative tests on phase change materials characterisation with differential scanning calorimeter. Appl. Energy 2013, 109, 415-420. [CrossRef]

32. ASTM C1872. Standard Test Method for Thermogravimetric Analysis of Hydraulic Cement. Astm Stand. 2018. [CrossRef]

33. ISO 11358-1. Plastics-Thermogravimetry (TG) of Polymers-Part 1: General Principles; International Organization for Standardization (ISO): Geneva, Switzerland, 2014.

34. ASTM E1131. Standard Test Method for Compositional Analysis by Thermogravimetry; ASTM International (ASTM): West Conshohocken, PA, USA, 2020. [CrossRef]

35. ASTM E2253. Standard Test Method for Temperature and Enthalpy Measurement Validation of Differential Scanning Calorimeters; ASTM International (ASTM): West Conshohocken, PA, USA, 2016. [CrossRef]

36. DIN 65583-04. Aerospace-Fibre Reinforced Materials - Determination of Glass Transition of Fibre Composites under Dynamic Load; Deutsches Institut fur Normung E.V. (DIN): Berlin, Germany, 1999. [CrossRef]

37. Gschwander, S.; Haussmann, T.; Hagelstein, G.; Solé, A.; Cabeza, L.F.; Diarce, G.; Hohenauer, W.; Lager, D.; Ristic, A.; Rathgeber, C.; et al. Standardization of PCM Characterization via DSC. In Proceedings of the 13th International Conference on Energy Storage, Greenstock, Trondheim, Norway, 19-21 May 2015.

38. Ravotti, R.; Fellmann, O.; Lardon, N.; Fischer, L.; Stamatiou, A.; Worlitschek, J. Synthesis and Investigation of Thermal Properties of Highly Pure Carboxylic Fatty Esters to Be Used as PCM. Appl. Sci. 2018, 8, 1069. [CrossRef]

39. Ouellette, R.J.; Rawn, J.D. 31-Lipids and Biological Membranes. In Organic Chemistry, 2nd ed.; Academic Press: Cambridge, MA, USA, 2018; pp. 1001-1032. ISBN 978-0-12-812838-1.

40. Acree, W.E.; Chickos, J.S. Phase Transition Enthalpy Measurements of Organic and Organometallic Compounds. In NIST Chemistry WebBook, NIST Standard Reference Database Number 69; Linstrom, P.J., Mallard, W.G., Eds.; National Institute of Standards and Technology (NIST): Gaithersburg, MD, USA, 2018.

41. Domalski, E.S.; Hearing, E.D. Condensed Phase Heat Capacity Data. In NIST Chemistry WebBook, NIST Standard Reference Database Number 69; Linstrom, P.J., Mallard, W.G., Eds.; National Institute of Standards and Technology (NIST): Gaithersburg, MD, USA, 2018.

42. Debenedetti, P.G. Metastable Liquids; Princeton University Press: Princeton, NJ, USA, 1996; Volume 1, ISBN 9780691085951.

43. Ventolà, L.; Ramírez, M.; Calvet, T.; Solans, X.; Cuevas-Diarte, M.A.; Negrier, P.; Mondieig, D.; van Miltenburg, J.C.; Oonk, H.A.J. Polymorphism of N-Alkanols: 1-Heptadecanol, 1-Octadecanol, 1-Nonadecanol, and 1-Eicosanol. Chem. Mater. 2002, 14, 508-517. [CrossRef]

44. Carlsson, B. Phase change behaviour of some latent heat storage media based on calcium chloride hexahydrate. Sol. Energy 2009, 83, 485-500. [CrossRef]

45. Schmit, H.; Rudaleviciene, D.; Rathgeber, C.; Hiebler, S. Calorimetric Investigation of Two Factors Influencing the Maximum Storage Capacity of Calcium Chloride Hexahydrate. Iop Conf. Ser. Mater. Sci. Eng. 2019, 660, 12076. [CrossRef] 
46. Wan, X.; Su, L.; Guo, B. Design and preparation of novel shapeable PEG/SiO2/AA shape-stabilized phase change materials based on double-locked network with enhanced heat storage capacity for thermal energy regulation and storage. Powder Technol. 2019, 353, 98-109. [CrossRef]

47. Chen, Z.; Cao, L.; Shan, F.; Fang, G. Preparation and characteristics of microencapsulated stearic acid as composite thermal energy storage material in buildings. Energy Build. 2013, 62, 469-474. [CrossRef]

48. Lin, Y.; Zhu, C.; Fang, G. Synthesis and properties of microencapsulated stearic acid/silica composites with graphene oxide for improving thermal conductivity as novel solar thermal storage materials. Sol. Energy Mater. Sol. Cells 2019, 189, 197-205. [CrossRef]

49. Wang, H.; Zhao, L.; Song, G.; Tang, G.; Shi, X. Organic-inorganic hybrid shell microencapsulated phase change materials prepared from $\mathrm{SiO} 2 / \mathrm{TiC}$-stabilized pickering emulsion polymerization. Sol. Energy Mater. Sol. Cells 2018, 175, 102-110. [CrossRef]

50. Li, C.; Yu, H.; Song, Y.; Liang, H.; Yan, X. Preparation and characterization of PMMA/TiO2 hybrid shell microencapsulated PCMs for thermal energy storage. Energy 2019, 167, 1031-1039. [CrossRef]

51. Arpagaus, C.; Berthold, M.; Eschmann, M. Bericht «WP-Feldmessung » (Auswertung verlängert bis Dez. 2019). Jahresbericht 2017 Energ. Schweiz 2019, 2018.

52. Höhlein, S.; König-Haagen, A.; Brüggemann, D. Thermophysical Characterization of MgCl2·6H2O, Xylitol and Erythritol as Phase Change Materials (PCM) for Latent Heat Thermal Energy Storage (LHTES). Materials (Basel) 2017, 10, 444. [CrossRef]

53. Gombás, Á.; Szabó-Révész, P.; Regdon, G.; Erős, I. Study of thermal behaviour of sugar alcohols. J. Anal. Calorim. 2003, 73, 615-621. [CrossRef]

54. Solé, A.; Neumann, H.; Niedermaier, S.; Cabeza, L.F.; Palomo, E. Thermal stability test of sugar alcohols as phase change materials for medium temperature energy storage application. Energy Procedia 2014, 48, 436-439. [CrossRef]

55. Fischer, L.J. N7 Phasenwechselmaterialien (PCM) für Latent-Wärmespeicher BT-VDI-Wärmeatlas: Fachlicher Träger VDI-Gesellschaft Verfahrenstechnik und Chemieingenieurwesen; Stephan, P., Kabelac, S., Kind, M., Mewes, D., Schaber, K., Wetzel, T., Eds.; Springer: Berlin/Heidelberg, Germany, 2019; pp. 1989-2008. ISBN 978-3-662-52989-8.

(C) 2020 by the authors. Licensee MDPI, Basel, Switzerland. This article is an open access article distributed under the terms and conditions of the Creative Commons Attribution (CC BY) license (http://creativecommons.org/licenses/by/4.0/). 